\title{
Removal of moisture from contaminated transformer oil in rectangular separators
}

\author{
Andrey Dmitriev ${ }^{1}$, Vadim Zinurov ${ }^{1}$, Dang Vinh $^{1}$, and Oksana Dmitrieva ${ }^{2, *}$ \\ ${ }^{1}$ Kazan State Power Engineering University, Krasnoselskaya str. 51, 420066 Kazan, Russia \\ ${ }^{2}$ Kazan National Research Technological University, Karl Marx str. 68, 420015 Kazan, Russia
}

\begin{abstract}
This paper deals with the removal of moisture from the contaminated transformer oil. Design of a rectangular separator and the results of water-oil emulsion separation are shown in this paper. The influence of different values of the separator height and the distance between the rows of elements on the emulsion separation efficiency was studied. In order to calculate the process of removing the moisture from transformer insulating oil, the multiphase Eulerian-Eulerian model "Volume of Fluid" with the number of phases equal to 2 was applied in ANSYS Fluent software package. $\mathrm{K}-\varepsilon$ turbulence model was used for the calculations. The results were obtained while solving the nonstationary issue. In the course of numerical simulation, the object of study was the transformer oil T-1500U, containing some water amount. The results of numerical simulation of water-oil emulsion separation in a rectangular separator are shown. In the course of numerical studies, it was found that the use of a rectangular separator in order to remove the moisture from transformer oil allows it to be purified from water by $99.99 \%$, providing that the geometrical dimensions of device are chosen correctly. The use of developed rectangular separator can be an alternative to the use of decanting tanks, various separators and other purification devices, which have extremely low rate of purification of contaminated spent oils. This separator allows purifying the transformer oil from water with a speed of $1-2 \mathrm{~m} / \mathrm{s}$ while the efficiency is equal to $99.99 \%$.
\end{abstract}

\section{Introduction}

As of today, the extension of transformer service life is an extremely urgent problem [13]. First, transformers are one of the main elements in the power supply system that allow converting the voltage of the electric current, and secondly, these devices belong to the

\footnotetext{
*Corresponding author: ja_deva@mail.ru
} 
most cost-intensive elements in the power supply system. That's why untimely failure of them can significantly negatively affect the energy supply and the financial status of enterprises [4-6].

The main failures of transformers are due to damage to the insulation system. It should be noted that insulating oil provides the greatest electrical strength in transformers. The low viscosity of oil allows it to penetrate into the solid insulation and remove the heat, transferring it to the cooling system. Stabilization of oils from oxidation allows them to work at high temperature and for a long time protects the insulation system from serious failures. However, when oxygen and water appear in the insulating oil, it is oxidized even under ideal conditions. The condition of insulating oil is also affected by various contaminants from solid materials of the transformer, which dissolve in the oil. As a result of the reactions that occur in the oil between unstable hydrocarbons, oxygen and water with the help of such accelerator as heat, the oil is oxidized, leading to its ageing and deterioration, which increases the load on the transformer insulation system. Insulation winding with $1 \%$ of moisture increases the ageing process by 10 times faster than with the moisture content of $0.1 \%$. Oxidation of the oil cannot be eliminated, but it can be controlled or slowed down through the maintenance process. One of the main provisions in the maintenance of the transformer is annual check of the oil. When the moisture is revealed, it is very important to eliminate it in a timely manner, as well as to maintain the low degree of moisture for insulation [7-10].

\section{Issues to be studied}

The purpose of this paper is to study the removal of moisture from the insulating transformer oil. A variety of technological operations are used to restore the spent oils. They involve the treatment of oils in order to remove the aging products and contaminants from them. The simplest and least cost-intensive methods of removing the water and various impurities from oils are physical methods of purification. The essence of these methods is the oil treatment, using gravitational, centrifugal, electric, magnetic and vibration forces. Also physical purification methods include various mass and heat exchange processes. Among them, the simplest method is sedimentation, based on the process of natural settling of mechanical particles, impurities and water under the action of gravitational forces. However, the main disadvantage of this method is the long duration period of the process, during which the spent oil is cleaned. Therefore, the development of new purification devices in order to remove various impurities and moisture from the oil is relevant [11-14].

The authors of this paper propose to use the developed rectangular separator in order to remove the moisture and impurities of different density than the transformer oil. The developed rectangular separator belongs to transformer oil regeneration devices and can be used at the enterprises of energy, electric and transformer stations and facilities, which use the transformer oil. A significant advantage of the developed separator is the spent oil treatment speed, which is equal to $1-2 \mathrm{~m} / \mathrm{s}$. For example, the same speed in decanting tanks is by 15-200 times lower [15-18].

The rectangular separator has a simple and compact design, consisting of several rows of U-shaped elements, assembled into a rectangular case $[19,20]$. There are inlet and outlet nozzles from both parallel sides of device, designed for the input of spent contaminated oil and the output of contaminants from the separator, respectively. Within the bottom of separator, along the middle row of U-shaped elements, there are two holes, serving for output of the purified transformer oil from device (Fig. 1). In order to increase the design strength, Ushaped elements are connected with each other by single transverse plate, located at the same distance from the lower and upper walls of the separator, which is fixed to the case of device. 


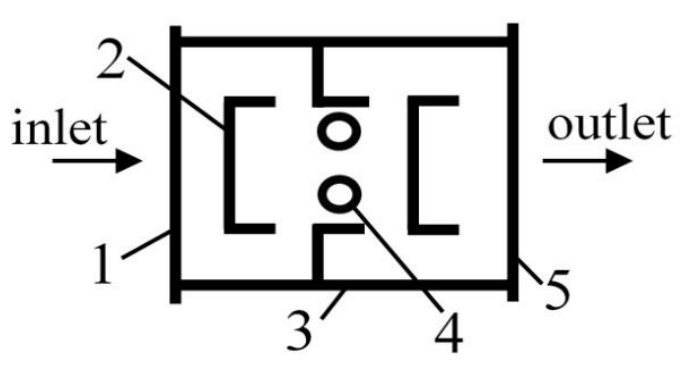

Fig. 1. Simplified model of rectangular separator: 1 - inlet nozzle, 2 - U-shaped elements, 3 - case of rectangular separator, 4 - holes for the outlet of purified transformer oil, 5 - outlet nozzle (top view).

Operation principle of this separator is based on the use of centrifugal force. When the contaminated transformer oil moves inside of the separator between U-shaped elements 2, the centrifugal force arises and divides the flow into different fractions, depending on their density. Also, additional separation of emulsion is facilitated by direct contact between the oil and the walls of separator's elements 2 . After the processes of contaminated oil separation into different fractions, the purified transformer oil moves into the executed holes 4 , while various impurities and water are removed through the outlet nozzle 5 (Fig. 1). Increase in the number of rows of U-shaped elements inside of the separator contributes to the increase in the efficiency of the contaminated oil purification.

\section{Methods of study}

In order to calculate the process of removing the moisture from transformer insulating oil, the multiphase Eulerian-Eulerian model "Volume of Fluid" with the number of phases equal to 2 was applied in ANSYS Fluent software package. In the course of numerical simulation, the object of study was the transformer oil T-1500U, containing some water amount. It should be noted that this type of transformer oil is widely used in Russia as the main electrical insulating material for filling the phase-shifting, furnace, grounding transformers. The volume ratios of water and oil T-1500U in the multiphase flow were taken equal to 0.5 each. Some other constant parameters were also set, namely: ambient temperature $t_{0}=20^{\circ} \mathrm{C}$; water density $\rho_{\mathrm{H} 20}=998.2 \mathrm{~kg} / \mathrm{m}^{3}$; water viscosity coefficient $\mathrm{H}_{2 \mathrm{O}}=$ $0.001003 \mathrm{~Pa} \mathrm{~s}$; transformer oil density $\rho_{\text {oil }}=883 \mathrm{~kg} / \mathrm{m}^{3}$; transformer oil viscosity coefficient oil $=0.0198 \mathrm{~Pa}$ s. In the course of study, the height $H$ of separator was changed in the range of 10-70 $\mathrm{mm}$, and the distance between the rows of U-shaped elements $h$ was also changed in the same range. When one parameter was changed, another was set to the base value. Base values were the following: separator height $H=30 \mathrm{~mm}$ and the distance between rows of U-shaped elements $h=30 \mathrm{~mm}$. K-E turbulence model was used for the calculations.

The results were obtained while solving the nonstationary issue. In the course of numerical simulation, the following boundary conditions were set: the inlet rate values ("velocity inlet") at the inlet nozzle of the rectangular separator was set in the range of $1-2 \mathrm{~m} / \mathrm{s}$; the atmospheric pressure was set equal to $10^{5} \mathrm{~Pa}$ at the outlet nozzle and within the holes for the outlet of purified transformer oil; and other boundaries had the "wall" condition Diameters of the outlet holes were taken equal to $2.25 \mathrm{~mm}$. Two values were changed in the course of study, namely, the distance between the rows of U-shaped elements and the height of the rectangular separator. When the height of the rectangular separator was changed, also the height of U-shaped elements changed proportionally.

The efficiency of transformer oil purification from moisture was determined by the following formula: 


$$
E=\left(1-C_{\text {oil-outlet }}\right) 100 \%,
$$

where $C_{\text {oil-outlet }}$ - oil volume ratio in the outlet nozzle.

Also the Reynolds number was determined in the course of studies as follows:

$$
\operatorname{Re}=\frac{W_{h} h}{v}
$$

where $W_{h}$ - multiphase flow rate within the narrowest places between U-shaped elements, $\mathrm{m} / \mathrm{s} ; h$ - distance between the rows of U-shaped elements, $\mathrm{m} ; v$ - kinematic viscosity of transformer oil, $\mathrm{m}^{2} / \mathrm{s}$.

\section{Results of studies and discussion of them}

The results of studies are shown graphically in Fig. 2-4. Numerical studies showed that the use of a rectangular separator in order to remove the moisture from transformer oil allows purifying it by $99.99 \%$ providing that the geometrical dimensions of device are chosen correctly.

The separation process of water-oil emulsion is shown in Fig. 2. Due to the effect of centrifugal force on the multiphase flow during its movement between U-shaped elements of the separator, and when the water-oil emulsion directly contacted with elements of device, liquid was divided into two fractions: water and oil. Due to the fact that the oil density is less than the water density, after the emulsion separation process completion, it rushed to the bottom of device and went out of the separator through special holes (Fig. 2).

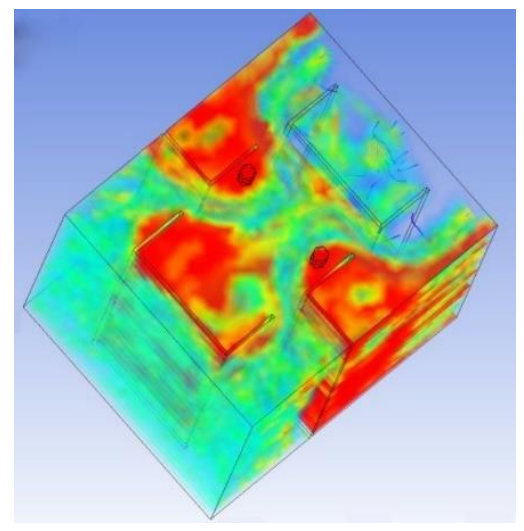

Fig. 2. Water-oil emulsion separation process inside of the rectangular separator.

In the process of studying the removal of moisture from the transformer oil by means of a rectangular separator, the movement of water-oil emulsion in the opposite direction was also considered. In other words, the contaminated oil was fed into the rectangular separator in such a way that the oil moved inside of U-shaped elements. It was assumed that such flow movement would increase the efficiency of the water-oil emulsion separation into fractions due to the formation of additional reverse flows inside of U-shaped elements, but the numerical calculations showed that the emulsion separation efficiency in such a way was by $10-20 \%$ lower than at the flow movement, shown in Fig. 1. Also, such movement contributes to an increase in the hydraulic resistance of the device due to the reverse flows inside of U-shaped elements. 


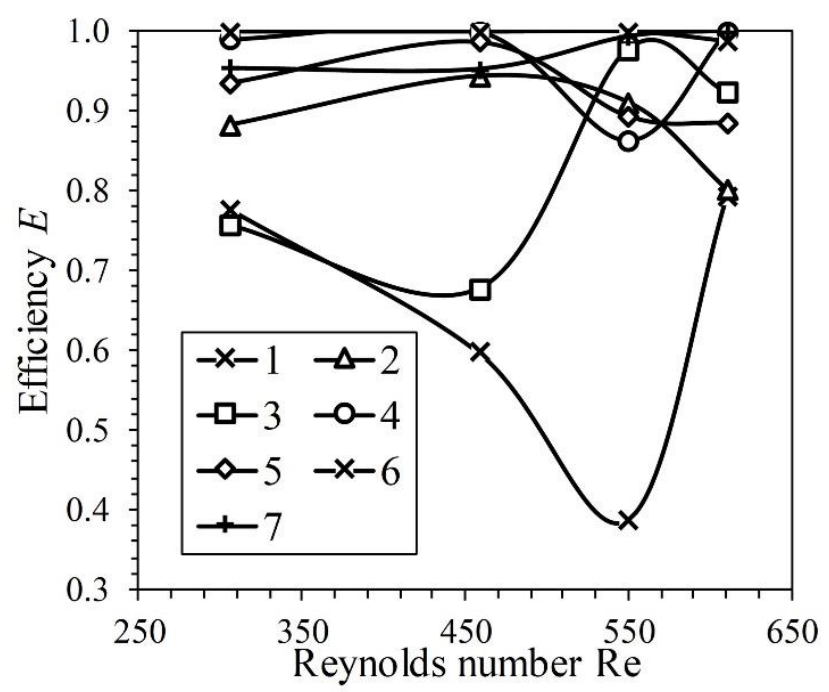

Fig. 3. Dependency of the water-oil emulsion separation efficiency inside of the separator on Reynolds number at the following height of device $H, \mathrm{~mm}: 1-10 ; 2-15 ; 3-20 ; 4-30 ; 5-50 ; 6-60 ; 7$ -70 .

The greatest efficiency of purifying the insulating transformer oil from moisture was observed at the studied height of device of $50 \mathrm{~mm}$. At this height, the efficiency of rectangular separator was $E=99.99 \%$. When the height of device was 70, 40, 30, 20, 15 and 10 $\mathrm{mm}$, the efficiency was on the average equal to $97,4 \%, 92,4 \%, 96,2 \%, 83,3 \%, 88,4 \%$ and $63.9 \%$ respectively. Purification of transformer oil with such efficiency is not satisfactory due to the fact that even an increase in moisture by less than $1 \%$ of the existing amount significantly affects its insulation characteristics. There is an option of several circles for contaminated transformer oil through the separator with a height of less than $50 \mathrm{~mm}$, however, this will extend the oil purification time period. Therefore, the most rational option is to increase the height of device up to $50 \mathrm{~mm}$. With an increase in the height of separator (more than $50 \mathrm{~mm}$ ), the efficiency also decreases due to the deviation of the vortex axis from the straight line (Fig. 3).

It should be noted that the change in Reynolds number, which is determined by the Eq. (2), is affected only by the flow rate within the narrowest places between U-shaped elements, while other parameters remain constant. Thus, the separation efficiency of the wateroil emulsion is high and at average is equal to $99.9 \%$, when the values of Reynolds number are in the range of 306-611, which correspond to the inlet flow rates $-1-2 \mathrm{~m} / \mathrm{s}$ for the separator with the height of $50 \mathrm{~mm}$ (Fig. 3).

The separation efficiency of the water-oil emulsion is significantly affected by the distance between the rows of U-shaped elements. As a rule, when this distance is increased up to $30 \mathrm{~mm}$, the efficiency of emulsion separation also increases. This situation is clearly observed on the 4th line in Fig. 4, corresponding to the inlet flow rate $w=2 \mathrm{~m} / \mathrm{s}$. 


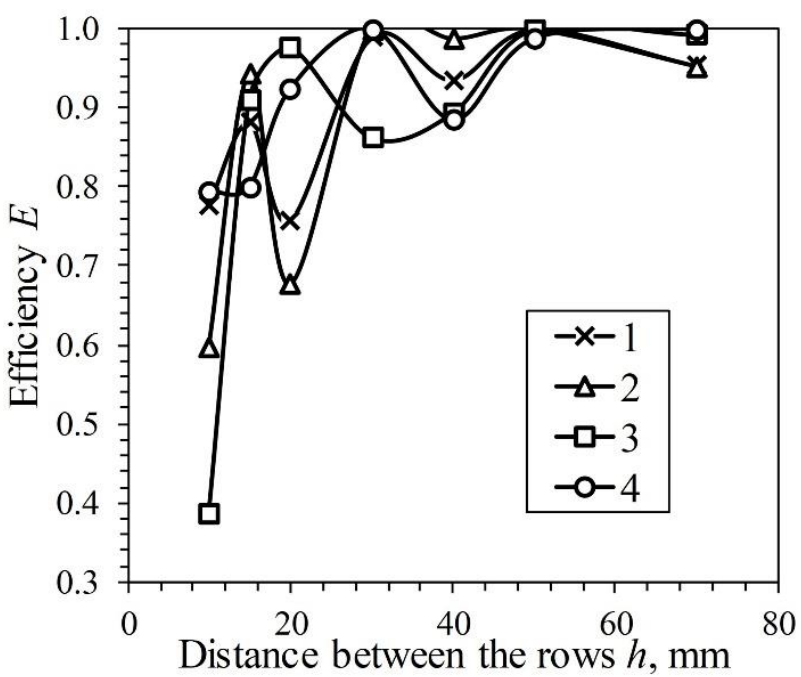

Fig. 4. Dependency of the water-oil emulsion separation efficiency inside of the separator on the distance between the rows of elements $h$ at the inlet flow rate $w, \mathrm{~m} / \mathrm{s}: 1-1 ; 2-1.5 ; 3-1.8 ; 4-2$.

Maximum efficiency of liquid separation, equal to $99.99 \%$, is achieved when the distance between the rows of U-shaped elements is $h=30 \mathrm{~mm}$, and for almost any inlet rate in the range of $1-2 \mathrm{~m} / \mathrm{s}$. However, when the inlet flow rate is $1.8 \mathrm{~m} / \mathrm{s}$, the separation efficiency is equal to $86.2 \%$, but it can be assumed that this value is fluctuating due to the fact that the separation efficiency is equal to $99.99 \%$ in both cases (Fig. 4) at the inlet rates of 1.7 and $1.9 \mathrm{~m} / \mathrm{s}$.

In the course of studies, it was found that the use of a rectangular separator, when the inlet rates of water-oil emulsion are in the range of 1-2 $\mathrm{m} / \mathrm{s}$, allows it to be purified from water by $99.99 \%$, providing that the height of device is $50 \mathrm{~mm}$ and the distance between the rows of U-shaped elements is equal to $30 \mathrm{~mm}$, respectively.

\section{Conclusion}

The use of developed rectangular separator can be an alternative to the use of decanting tanks, various separators and other purification devices, which have extremely low rate of purification of contaminated waste oils. The developed separator allows purifying the transformer oil from water with a speed of $1-2 \mathrm{~m} / \mathrm{s}$ while the efficiency is equal to $99.99 \%$. The significant advantages of the rectangular separator are the following: compactness, small dimensions, high efficiency, easy to use and manufacture.

The study was carried out with funding from the grant of the President of the Russian Federation No. MK-4522.2018.8.

\section{References}

1. O.S. Dmitrieva, G.R. Patrakova, A.V. Dmitriev, 2017 International Conference on Industrial Engineering, Applications and Manufacturing (ICIEAM), 1-4 (2017) https://doi.org/10.1109/ICIEAM.2017.8076305

2. S. Prasanna Raj Yadav, C.G. Saravanan, R. Vallinayagam, S. Vedharaj, W.L. Roberts, Energy Convers. Manage 96, 490-498 (2015) https://doi.org/10.1016/j.enconman.2015.02.051 
3. G. Jiang, W. Fu, S. Shu, Z. Zhang, S. Zhang, Y. Zhang, X. Zhang, F. Dong, X. Lv, J. Hazard. Mater. 367, 658-667 (2019) https://doi.org/10.1016/j.jhazmat.2019.01.018

4. W. Bengang, H. Hua, L. Honglei, W. Nannan, D. Mingqiu, Energy Procedia 100, 556560 (2016) https://doi.org/10.1016/j.egypro.2016.10.218

5. P.A. Córdoba, E. Dari, N. Silin, Appl. Therm. Eng. 148, 897-906 (2019) https://doi.org/10.1016/j.applthermaleng.2018.11.098

6. M.I. Hasan, Engineering Science and Technology 20(2), 502-510 (2017) https://doi.org/10.1016/j.jestch.2016.12.003

7. A.H. Mohammed Abdul-Karim, M.A. Kadhum, Journal of Engineering 16(4), 5799$5810(2010)$

8. V.K. Kozlov, M.S. Garifullin, J. Eng. Appl. Sci. 11(14), 3042-3046 (2016) https://doi.org/10.3923/jeasci.2016.3042.3046

9. N.D. Yakimov, O.S. Dmitrieva, Russ. Electr. Engin. 89(6), 367-370 (2018) https://doi.org/10.3103/S1068371218060123

10. A. Ortiz, F. Delgado, F. Ortiz, I. Fernández, A. Santisteban, Appl. Therm. Eng. 144, 797-803 (2018) https://doi.org/10.1016/j.applthermaleng.2018.08.049

11. X. Lv, D. Tian, Y. Peng, J. Li, G. Jiang, Appl. Surf. Sci. 466, 937-945 (2019) https://doi.org/10.1016/j.apsusc.2018.10.110

12. C.F. Medina-Sandoval, J.A. Valencia-Dávila, M.Y. Combariza, C. Blanco-Tirado, Fuel 231, 297-306 (2018) https://doi.org/10.1016/j.fuel.2018.05.066

13. F.Sh. Khafizov, V.G. Afanasenko, I.F. Khafizov, A.Sh. Khaibrakhmanov, E.V. Boev, Chem. Pet. Eng. 44, 425-28 (2008) https://doi.org/10.1007/s10556-008-9081-z

14. A.V. Dmitriev, I.N. Madyshev, O.S. Dmitrieva, Proceedings of the 4th International Conference on Industrial Engineering. ICIE 2018. Lecture Notes in Mechanical Engineering, Springer, 1903-1909 (2019) https://doi.org/10.1007/978-3-319-95630-5_204

15. S.K. Shukla, P. Shukla, P. Ghosh, Appl. Math. Model. 37, 5774-89 (2013) https://doi.org/10.1016/j.apm.2012.11.019

16. A. Krasinski, Filtr. Sep. 51(6), 22-28 (2014) https://doi.org/10.1016/S00151882(14)70224-1

17. M. Santander, R.T. Rodrigues, J. Rubio, Colloids Surf. A. 375(1-3), 237-244 (2011) https://doi.org/10.1016/j.colsurfa.2010.12.027

18. V.K. Rajak, I. Singh, A. Kumar, A. Mandal, Pet. Sci. Technol. 34(11-12), 1026-1032 (2016) https://doi.org/10.1080/10916466.2016.1181654

19. A.V. Dmitriev, V.E. Zinurov, O.S. Dmitrieva, IOP Conf. Ser.: Mater. Sci. Eng. 451, 012211 (2018) https://doi.org/10.1088/1757-899X/451/1/012211

20. A.V. Dmitriev, V.E. Zinurov, O.S. Dmitrieva, MATEC Web Conf. 224, 02073 (2018) https://doi.org/10.1051/matecconf/201822402073 